\title{
ACOMPANHAMENTO ASSISTENCIAL DO PRÉ- NATAL AS GESTANTES DURANTE A PANDEMIA DE COVID-19
}

\author{
PRENATAL CARE FOR PREGNANT WOMEN DURING THE COVID-19 \\ PANDEMIC
}

\author{
Luana Lins de Oliveira ${ }^{1}$ \\ Ocilma Barros de Quental² \\ Geane Silva Oliveira ${ }^{3}$ \\ Jakeline Pamplona Sarmento ${ }^{4}$ \\ Laergyla Maria Oliveira Dionisio ${ }^{5}$ \\ Macerlane de Lira Silva ${ }^{6}$
}

RESUMO: O período gestacional traz transformações fisiológicas na mulher, com isso, está apresenta maior vulnerabilidade, portanto, a execução de uma assistência pré-natal de forma adequada e qualificada promovem maior conforto e segurança à gestante. O pré-natal possibilita que a gestação progrida de forma favorável para a mãe e o feto, proporcionando uma melhor qualidade de vida materno- infantil. Em 2020, com a pandemia de Covid-19, os cuidados durante o pré-natal intensificaramse ainda mais com a inclusão de mulheres grávidas como grupo de risco para infecção por Sars-Cov-2.Trata- se de uma revisão integrativa da literatura, realizada em 2021, utilizando os descritores "GRAVIDEZ", "PRÉ- NATAL" e "COVID-19", que devem ser verificados pelo DeCS (Descritores em Ciências da Saúde), as bases de dados utilizadas foram: LILACS (Literatura Latino Americana e do Caribe em Ciências da Saúde), SciELO (ScientificElectronic Library onLine) e BVS (Biblioteca Virtual de Saúde).Os critérios para a seleção dos estudos foram artigos publicados nos últimos 07 (sete) anos, de 2015 a 2021, artigos nacionais e internacionais, disponíveis em português, trabalhos originais, completos, grátis para acesso e que

\footnotetext{
1 Luana Lins de Oliveira ${ }^{1}$ - Graduanda do curso de Enfermagem da Faculdade Santa Maria de Cajazeiras.Iuanalins29072000@gmail.com.

2 Ocilma Barros de Quental ${ }^{2}$ - Enfermeira. Doutora em ciências da Saúde. Docenteda Faculdade Santa Maria - FSM.Dra.quental@gmail.com.

${ }^{3}$ Geane Silva Oliveira ${ }^{3}$ - Enfemeira. Mestre em enfermagem. Docenteda Faculdade Santa Maria FSM.Geane32.silva@gmail.com.

${ }^{4}$ Jakeline Pamplona Sarmento ${ }^{4}$ - Graduanda do curso de Enfermagem da Faculdade Santa Maria de Cajazeiras.Jakelinepam34@gmail.com.

${ }^{5}$ Laergyla Maria Oliveira Dionisio ${ }^{5}$ - Graduanda do curso de Enfermagem da Faculdade Santa Maria de Cajazeiras. Laergyla124@outlook.com.

${ }^{6}$ Macerlane de Lira Silva ${ }^{6}$ - Enfermeiro. Mestre em Saúde Coletiva. Docente da Faculdade Santa Maria - FSM.marcerlane@hotmail.com.
} 
responderam a seguinte questão norteadora: quais são os efeitos da pandemia de COVID- 19 para as gestantes? E como isso afeta o acompanhamento pré-natal nas unidades básicas de saúde? O critério de exclusão foram artigos que não condizem com o estudo proposto. Os artigos selecionados sucederam para a elaboração dos resultados da pesquisa. Os resultados expostos evidenciam que a assistência prénatal é, e continua sendo, de extrema importância tanto para saúde materna quanto para a saúde fetal e perinatal, com enfrentamento a pandemia, a assistência contribui para o fortalecimento dos cuidados e precauções das mulheres em período gravídico para evitar o contágio de COVID-19, mostrando a relevância da qualificação da assistência pré-natal e evidenciando a significância dos profissionais de saúde e da atenção básica.

Descritores: Gravidez. Pré-natal. COVID-19.

ABSTRACT: The gestational period brings physiological changes in women, with this, they are more vulnerable, therefore, the implementation of adequate and qualified prenatal care promote greater comfort and safety for pregnant women. Prenatal care allows the pregnancy to progress in a favorable way for the mother and fetus, providing a better quality of maternal-infant life. In 2020, with the Covid-19 pandemic, prenatal care was further intensified with the inclusion of pregnant women as a risk group for Sars-Cov-2 infection. This is an integrative literature review, carried out in 2021, using the descriptors "PREGNANCY", "PRENATAL" and "COVID-19", which must be verified by DeCS (Descriptors in Health Sciences), the bases of data used were: LILACS (Latin American and Caribbean Literature on Health Sciences), SciELO (ScientificElectronic Library onLine) and VHL (Virtual Health Library). The criteria for the selection of studies were articles published in the last 07 (seven) years, from 2015 to 2021, national and international articles, available in Portuguese, original, complete works, free to access and that answered the following guiding question: what are the effects of the COVID-19 pandemic for pregnant women? And how does this affect prenatal care in basic health units? The exclusion criteria were articles that do not match the proposed study. The selected articles succeeded in preparing the research results. The results presented show that prenatal care is, and continues to be, extremely important for both maternal and fetal and perinatal health, with the fight against the pandemic, the assistance contributes to the strengthening of care and precautions for women during this period. pregnancy to prevent the spread of COVID-19, showing the relevance of qualifying prenatal care and highlighting the significance of health professionals and primary care.

Descriptors: Pregnancy. Prenatal. COVID-19. 


\section{INTRODUÇÃO}

Após a implementação do Programa de Assistência Integral à Saúde da Mulher (PAISM), várias questões relacionadas ao bem-estar e a qualidade de vida das mulheres foram percebidas, e a partir disso, o Ministério da Saúde sistematizou uma linha prioritária de cuidados à mulher, com o intuito de melhorar a saúde reprodutiva, reduzir as mortes por causas evitáveis e combater à violência contra o público feminino. Com o intuito de melhorar a qualidade reprodutiva, foi criado um programa voltado ao pré-natal, lançado em junho de 2000, com a estratégia de garantir uma melhor assistência do atendimento, acesso, cobertura e qualidade durante a gestação, parto, puerpério e cuidados ao recém-nascido (BRASIL, 2002).

A gestação é um momento singular na vida de qualquer mulher, por isso, a realização de uma assistência pré-natal qualificada é de extrema importância para proporcionar segurança para a mãe e seu filho. Esse acompanhamento visa garantir a evolução da gravidez de forma saudável, sem riscos para a gestante ou para o feto, detectando possíveis doenças de forma precoce e promovendo melhor qualidade de vida. Os serviços fornecidos durante a assistência pré-natal incluem: consultas, vacinação, exames laboratoriais e ultrassonografia, além disso, também são dadas orientações quanto à higiene, saúde psicológica, alimentação e medicações recomendadas durante a fase gestacional (ESPOSTI, 2020).

A cobertura de atendimento pré-natal no Brasil aumentou, porém, de acordo com os procedimentos preconizados pelo Ministério da Saúde, foram encontrados problemas com a qualidade da assistência. Em diferentes circunstâncias, a taxa de adequação do processo pré-natal é baixa e os níveis podem levar a consequências indesejáveis, como o nascimento de bebês prematuros e aumento da mortalidade materna e perinatal. (MEDEIROS, 2019).

Sabe-se que a qualidade da assistência pré-natal afeta diretamente os indicadores de saúde e ajuda a reduzir a morbimortalidade das gestantes e no período perinatal. Porém, o aumento da incidência de casos de sífilis congênita e o 
fato da causa mais comum de morte materna ser a hipertensão arterial sugerem que: embora a cobertura pré-natal venha aumentando, a qualidade dessa assistência ainda precisa de melhorias (LIVRAMENTO, 2019).

Estrela (2020) apresenta que devido a propagação do novo coronavírus, SARS-COV-2, patógeno da covid- 19, as mulheres grávidas tornaram-se mais vulneráveis. Levando em consideração a gestação e as complicações fetais, é necessário refletir sobre as condições da gravidez durante a pandemia de Covid-19 e a importância do cuidado profissional, principalmente do enfermeiro, para superar os inúmeros desafios que permeiam esse cenário. A Organização Mundial da Saúde (OMS) classifica as mulheres grávidas como um grupo de alto risco para Covid-19, em virtude a probabilidade de morbidade e mortalidade.

De acordo com Silva (2020), muitas mulheres se preocupam com os problemas que podem ocorrer durante a gravidez e o parto, como a possibilidade de transmissão vertical do vírus. De acordo com os últimos estudos, mulheres grávidas com condições graves que desenvolvem infecção por Sars-Cov-2 agregadas a outras comorbidades têm maior probabilidade de serem submetidas à cesariana de emergência ou parto prematuro.

Entretanto, na maioria das gestantes infectadas, os sintomas são leves, como febre e tosse seca. No entanto, em mulheres na segunda metade da gravidez, outros sintomas de menor intensidade também podem ocorrer, como: fadiga, dificuldade para respirar, diarreia, congestão nasal e rinite (ESTRELA, 2020).

Ressalta-se, ainda, que devido às constantes mudanças na rede de saúde, os enfermeiros enfrentam desafios crescentes na gestão e na assistência de enfermagem, e novas estratégias precisam ser planejadas para atender às necessidades das mulheres em período gravídico. Algumas dessas estratégias são: reorganização dos fluxos da rede; acompanhamento e orientação virtual; triagem de classificação de risco; consultas de pré-natal de rotina e procedimentos para gestantes com sintomas gripais devem ser adiados por 14 dias (BRASIL, 2020).

Desse modo, para assistir esse público de forma qualificada diante da pandemia de COVID-19, é necessário reorganizar o processo de atendimento do pré-natal, priorizando as medidas de controle e reduzindo os riscos de infecção, sem afetar ou desamparar as gestantes. Em virtude do que foi mencionado, indaga- se: 
quais são os efeitos da pandemia de COVID- 19 para a gestante e como isso afeta o acompanhamento pré-natal nas unidades básicas de saúde?

Esta pesquisa mostra a relevância de uma assistência qualificada às gestantes em meio ao cenário atual e como um pré-natal bem assistido pode prevenir doenças evitáveis e proporcionar uma experiência gestacional mais agradável, destacando o papel do enfermeiro e as principais linhas de cuidado.

\section{MÉTODO}

Trata-se de uma revisão integrativa da literatura, sucedendo- se de pesquisas que coletam dados de fontes auxiliares por meio de levantamento bibliográfico.

De acordo com Sousa et al., (2017), a revisão integrativa da literatura é um tipo de pesquisa que consiste em métodos investigativos, ou seja, é baseada na experiência do autor durante a execução de uma revisão integrativa, portanto, os resultados são apresentados de forma qualitativa. A revisão integrativa da literatura é dividida em seis fases, que são elas:

- $\quad 1^{\circ}$ fase: elaboração da pergunta norteadora

- $\quad 20$ fase: busca ou amostragem na literatura

- $\quad 3$ fase: coleta de dados

- $\quad$ 4ํ fase: análise crítica dos estudos incluídos

- $\quad 5^{\circ}$ fase: discussão dos resultados

- 6 fase: apresentação da revisão integrativa

Todas essas etapas foram percorridas para elaboração deste estudo, que teve como eixo norteador a seguinte questão: COMO SE APRESENTA A ASSISTÊNCIA PRÉ-NATAL DURANTE A PANDEMIA COVID-19?

A busca bibliográfica ocorreu através das bases de dados SCIELO, LILACS e Biblioteca Virtual de Saúde (BVS), pelo intercruzamento dos seguintes descritores: "Gravidez", "Pré-natal" e "COVID-19". Os critérios de inclusão consistiram em artigos publicados nos últimos sete anos, disponíveis na íntegra e acesso gratuito, em idioma português, que abordassem o objetivo da pesquisa. Foram excluídas 
editoriais, cartas ao editor, cartilhas, tese, dissertação artigos repetidos e estudos que não correspondessem à temática relevante do estudo.

Desse modo, os artigos selecionados sintetizaram os resultados, os quais estão expostos na tabela de forma organizada e descritiva, para que possam ser realizadas análises críticas e sistemáticas e a literatura possa ser discutida, observada, descrita e classificada diante a exposição das informações.

\section{RESULTADOS}

A assistência pré-natal desempenha um papel importante na prevenção e/ou detecção precoce das doenças maternas e fetais, acompanha o desenvolvimento do bebê para que a gestação ocorra da maneira mais saudável tanto para o feto quanto para a gestante, reduzindo assim, riscos e possíveis agravos.

A pesquisa fez uma análise dessa assistência prestada às mulheres grávidas em meio à pandemia de Covid- 19 com o intuito de fazer um levantamento sobre as dificuldades e vulnerabilidades encontradas e quais os caminhos necessários para a realização de um pré-natal qualificado diante um cenário atípico.

No estudo foram selecionados sete (07) artigos que contemplam e relacionam- se com o objetivo e as adversidades encontradas na pesquisa: "ACOMPANHAMENTO ASSISTENCIAL DO PRÉ- NATAL AS GESTANTES DURANTE A PANDEMIA DE COVID-19". O quadro a seguir traz a concepção dos autores em relação ao tema proposto. 
Quadro 1 - Análise dos Resultados.

\begin{tabular}{|c|c|c|c|c|}
\hline $\begin{array}{l}\text { AUTORES } \\
\text { ANO }\end{array}$ & TÍTULO & $\begin{array}{l}\text { LOCAL DE } \\
\text { PUBLICAÇÃO }\end{array}$ & OBJETIVO & $\begin{array}{c}\text { ADVERSIDADES } \\
\text { ENCONTRADAS NO } \\
\text { ACOMPANHAMENTO } \\
\text { A GESTANTES } \\
\text { DURANTE A } \\
\text { PANDEMIA } \\
\end{array}$ \\
\hline $\begin{array}{l}\text { CARDOSO et } \\
\text { al., } 2020\end{array}$ & $\begin{array}{l}\text { A saúde } \\
\text { materno-infantil } \\
\text { no contexto da } \\
\text { pandemia de } \\
\text { COVID-19: } \\
\text { evidências, } \\
\text { recomendações } \\
\text { e desafios. }\end{array}$ & $\begin{array}{l}\text { Rev. Brasileira } \\
\text { de Saúde } \\
\text { Materno } \\
\text { Infantil }\end{array}$ & $\begin{array}{l}\text { O estudo tem } \\
\text { como finalidade } \\
\text { expor, no } \\
\text { contexto da } \\
\text { pandemia de } \\
\text { COVID-19, as } \\
\text { principais } \\
\text { evidências, } \\
\text { recomendações } \\
\text { e desafios para } \\
\text { a saúde } \\
\text { materno- } \\
\text { infantil. }\end{array}$ & $\begin{array}{l}\text { Existem vários desafios } \\
\text { que perpetuam durante } \\
\text { uma gravidez em } \\
\text { cenário pandêmico, } \\
\text { como: a possibilidade } \\
\text { da transmissão vertical } \\
\text { do vírus, insegurança } \\
\text { em relação a } \\
\text { amamentação a saúde } \\
\text { prejudicando a saúde } \\
\text { do binômio mãe-filho- e } \\
\text { uma maior } \\
\text { vulnerabilidade àde à } \\
\text { mulher que pode levar } \\
\text { consequências a sua } \\
\text { saúde mental. }\end{array}$ \\
\hline $\begin{array}{l}\text { ESTRELA et al., } \\
2020\end{array}$ & $\begin{array}{lr}\text { Gestantes } & \text { no } \\
\text { contexto } & \text { da } \\
\text { pandemia } & \text { da } \\
\text { Covid-19: } & \\
\text { reflexões } & \text { e } \\
\text { desafios. } & \end{array}$ & $\begin{array}{l}\text { PHYSIS: } \\
\text { Revista de } \\
\text { Saúde Coletiva }\end{array}$ & $\begin{array}{l}\text { Destaca } \\
\text { papel } \\
\text { fundamental do } \\
\text { enfermeiro na } \\
\text { atenção } \\
\text { primária. }\end{array}$ & $\begin{array}{l}\text { Evidencia que à medida } \\
\text { que as redes de saúde } \\
\text { continuam mudando } \\
\text { diante ao cenário } \\
\text { pandêmico, os } \\
\text { enfermeiros enfrentam } \\
\text { desafios crescentes na } \\
\text { gestão e na assistência } \\
\text { de enfermagem, } \\
\text { fazendo necessário o } \\
\text { desenvolvimento de } \\
\text { novas estratégias para } \\
\text { atender a às } \\
\text { necessidades das } \\
\text { gestantes. }\end{array}$ \\
\hline $\begin{array}{l}\text { MASCARENHAS } \\
\text { et al., } 2020\end{array}$ & $\begin{array}{l}\text { COVID-19 e a } \\
\text { produção de } \\
\text { conhecimento } \\
\text { sobre as } \\
\text { recomendações } \\
\text { na gravidez: } \\
\text { revisão de } \\
\text { escopo. }\end{array}$ & $\begin{array}{l}\text { Rev. Latino- } \\
\text { Americana de } \\
\text { Enfermagem }\end{array}$ & $\begin{array}{l}\text { Realiza o } \\
\text { mapeamento } \\
\text { da produção } \\
\text { acerca do } \\
\text { conhecimento } \\
\text { sobre as } \\
\text { recomendações } \\
\text { assistenciais à } \\
\text { gestante diante } \\
\text { ao novo } \\
\text { coronavírus. }\end{array}$ & $\begin{array}{l}\text { Após o vírus circular em } \\
\text { todo o mundo e ser } \\
\text { declarada pandemia } \\
\text { mundial, instalou- se } \\
\text { um novo desafio: } \\
\text { ofertar os serviços de } \\
\text { saúde a toda } \\
\text { população. }\end{array}$ \\
\hline $\begin{array}{l}\text { AMORIM et al., } \\
2021\end{array}$ & $\begin{array}{l}\text { COVID-19 } \\
\text { Gravidez. }\end{array}$ & $\begin{array}{l}\text { Rev. Brasileira } \\
\text { de Saúde } \\
\text { Materno } \\
\text { Infantil }\end{array}$ & $\begin{array}{lr}\text { O } & \text { presente } \\
\text { estudo abrange } \\
\text { a perspectiva } \\
\text { da gravidez } \\
\text { durante } \quad \text { a } \\
\text { pandemia de }\end{array}$ & 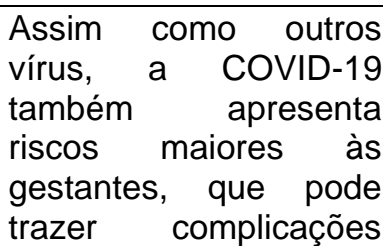 \\
\hline
\end{tabular}




\begin{tabular}{|c|c|c|c|c|}
\hline & & & COVID-19. & $\begin{array}{l}\text { para a mãe e para o } \\
\text { feto, como: trabalho de } \\
\text { parto prematuro, } \\
\text { ruptura prematura de } \\
\text { membranas, restrição } \\
\text { de crescimento fetal, } \\
\text { abortamento, pré- } \\
\text { eclâmpsia } \\
\text { pneumonia. }\end{array}$ \\
\hline $\begin{array}{l}\text { OLIVEIRA et al., } \\
2021\end{array}$ & $\begin{array}{ll}\text { Recomendações } \\
\text { para assistência } \\
\text { perinatal } & \text { no } \\
\text { contexto } & \text { da } \\
\text { pandemia } & \text { de } \\
\text { COVID- 19. } & \end{array}$ & $\begin{array}{l}\text { Rev. Brasileira } \\
\text { de Saúde } \\
\text { Materno } \\
\text { Infantil }\end{array}$ & $\begin{array}{l}\text { Elenca } \\
\text { orientações e } \\
\text { recomendações } \\
\text { a assistência } \\
\text { perinatal - que } \\
\text { envolve todo o } \\
\text { ciclo } \\
\text { gestacional, o } \\
\text { trabalho de } \\
\text { parto, } \\
\text { nascimento, } \\
\text { puerpério e os } \\
\text { cuidados ao } \\
\text { recém-nascido. }\end{array}$ & $\begin{array}{lr}\text { Espera } & \text { novas } \\
\text { evidências } & \text { e } \\
\text { atualizações cientificas } \\
\text { sobre a temática para } \\
\text { que ocorra uma } \\
\text { assistência perinatal } \\
\text { adequada, segura e } \\
\text { livre de possíveis } \\
\text { agravos no contexto da } \\
\text { pandemia por COVID }\end{array}$ \\
\hline $\begin{array}{l}\text { SILVA et al., } \\
2021\end{array}$ & $\begin{array}{lr}\text { Gravidez, parto } \\
\text { e puerpério na } \\
\text { pandemia: } \\
\text { múltiplos } \\
\text { sentidos } \\
\text { risco. }\end{array}$ & $\begin{array}{l}\text { Rev. } \\
\text { Horizontes } \\
\text { Antropológicos }\end{array}$ & $\begin{array}{lr}\text { A partir } & \text { da } \\
\text { pesquisa } & \text { do } \\
\text { risco } & \text { da } \\
\text { gravidez } & \text { e } \\
\text { nascimento } & \\
\text { durante } & \text { a } \\
\text { pandemia, } & \text { o } \\
\text { estudo tem a } & \\
\text { finalidade de } \\
\text { responder } \\
\text { questões sobre } \\
\text { a } \\
\text { vulnerabilidade } \\
\text { física } \\
\text { gestantes das } \\
\text { puérperas, o } \\
\text { conceito de } \\
\text { infecção por } \\
\text { COVID-19 e a } \\
\text { problemática da } \\
\text { mortalidade } \\
\text { materna. }\end{array}$ & $\begin{array}{l}\text { Os desafios trazidos } \\
\text { pela nova doença } \\
\text { afetam diretamente as } \\
\text { grávidas, parturientes e } \\
\text { puérperas, e nesse } \\
\text { contexto, os riscos de } \\
\text { complicações por } \\
\text { infecção de COVID-19 } \\
\text { são mais suscetíveis a } \\
\text { estes grupos. }\end{array}$ \\
\hline $\begin{array}{l}\text { STOFEl et al., } \\
2021\end{array}$ & $\begin{array}{lr}\text { Atenção } & \\
\text { perinatal } & \text { na } \\
\text { pandemia } & \text { da } \\
\text { COVID-19: } & \\
\text { análise } & \text { de } \\
\text { diretrizes } & \text { e } \\
\text { protocolos } & \\
\text { nacionais. } & \end{array}$ & $\begin{array}{l}\text { Rev. Brasileira } \\
\text { de } \quad \text { Saúde } \\
\text { Materno } \\
\text { Infantil }\end{array}$ & \begin{tabular}{lr} 
Analisa & o \\
protocolo & \\
proposto & pelo \\
Ministério & da \\
Saúde & na \\
atenção & \\
perinatal & no \\
processo & de \\
cuidado & e \\
atendimento de \\
mulheres diante \\
\multicolumn{2}{l}{ a pandemia. }
\end{tabular} & $\begin{array}{l}\text { Embora exista um } \\
\text { déficit na adequação ao } \\
\text { contexto em saúde no } \\
\text { Brasil, as maiorias das } \\
\text { recomendações } \\
\text { harmonizam com os } \\
\text { princípios para uma } \\
\text { experiência agradável } \\
\text { na Peri natalidade. }\end{array}$ \\
\hline
\end{tabular}

Fonte: dados da pesquisa, 2021. 


\section{DISCUSSÃO}

De acordo com Livramento (2019), no que se refere a assistência pré- natal, é importante iniciá- la o mais rápido possível, realizar os exames recomendados, detectar e tratar possíveis alterações e evitar riscos que possam afetar o binômio mãe-filho. É importante também que a gestante receba orientação e apoio quanto às mudanças ocasionadas pela gravidez, alimentação adequada, atividades físicas e diárias, uso de substâncias nocivas, sinais e sintomas de risco, alívio de desconfortos, amamentação, seus direitos e orientações locais.

Segundo Livramento (2020), perante a atual pandemia de coronavírus, SARSCOV-2, agente etiológico da Covid-19, que propagou- se rapidamente no mundo inteiro, vulnerabilizando, além de outros grupos, as mulheres grávidas, fez- se necessário refletir sobre estar grávida diante de um cenário atípico.

Diante a isso que foi exposto, destaca- se os seguintes tópicos:

\section{Vulnerabilidades das gestantes como grupo de risco para Covid-19}

Diante os primeiros estudos acerca dos desdobramentos do SARS-CoV-2 na gestação, observou- se que mulheres grávidas podem apresentar uma maior vulnerabilidade e desenvolver a forma mais agressiva da doença. $E$, tendo isso em vista, em março de 2020, o Ministério da Saúde incluiu as gestantes como grupo de risco para Covid-19, levando em conta as particularidades que a gravidez traz para a mulher, principalmente relacionada às alterações fisiológicas e imunológicas. (MASCARENHAS et al., 2020).

De acordo com Silva (2020), e sua concepção dos inúmeros sentidos de riscos da gravidez, parto e puerpério na pandemia, a inclusão das gestantes como grupo de risco trouxe um conceito-chave para gerenciar e prevenir consequências 
adversas. De modo que essa designação simboliza um planejamento político de extrema importância que proporciona e objetiva a saúde materna e assegura seus direitos previstos por lei.

A gravidez não eleva os riscos da doença, porém, gestantes que apresentam comorbidades como: sobrepeso, diabetes, hipertensão e imunodeficiências podem evoluir para um quadro mais grave se forem infectadas. (OLIVEIRA, 2021).

Em virtude disso, o Ministério da Saúde (2020) incluiu as gestantes como grupo de risco da Covid-19 a fim de evitar agravamentos para esta população. $\mathrm{O}$ acompanhamento às mulheres grávidas, no contexto brasileiro, deixa a desejar e ainda não é o ideal, por isso que designá-las como ameaçadas e mais vulneráveis ao vírus traz uma assistência mais completa, priorizando a saúde materna, protegendo o binômio mãe-filho e propiciando uma gravidez segura.

Durante a gravidez, além das alterações hormonais, ocorrem também fatores relacionados à maternagem - que é definida como um vínculo afetivo, que envolve proteger e cuidar dos filhos de forma afetuosa e cuidadosa, que se desenvolve ao longo da vida como uma característica da mulher. Portanto, presenciar uma pandemia enquanto se está gestante, traz sentimentos de angústia, preocupação, ansiedade, dúvidas e inseguranças, visto que ainda não há consenso entre os estudos sobre a relação entre a gravidade da doença e esse período (ESTRELA, 2020).

\section{Papel do enfermeiro da APS (Atenção Primária a Saúde) na assistência pré- natal durante o enfrentamento a pandemia}

Diante a situação epidemiológica de infecção por Covid-19, devem ser instituídos nos serviços de atendimento as gestantes uma triagem e avaliação de alguns sintomas e fatores de riscos. Torna-se indescritível garantir às mulheres cuidados profissionais em medicina obstétrica e fetal, neonatal, incluindo saúde mental e apoio psicossocial para gestantes e puérperas (CARDOSO et al., 2020). 
Estrela (2020), destacou que o cenário pandêmico desafiou a assistência da enfermagem na Atenção Primária a Saúde, enfatizando as adversidades encontradas e as novas estratégias a serem elaboradas nesse contexto. Salientou também que o ambiente de atuação do enfermeiro da APS vai além do cuidado e atendimento recomendados durante o pré-natal, incluindo orientações, informações e medidas preventivas contra a Covid-19.

Os profissionais de saúde procuraram maneiras de adaptação para proteger os pacientes, a si próprios e aos recursos de saúde. Os sistemas de saúde do Brasil e de outros países restringiram consultas e procedimentos eletivos. Mediante a um planejamento criterioso, mudanças devem ser definidas neste cuidado, visando reduzir o contato dos pacientes, o tempo de permanência no consultório, clínica ou hospital, sem afetar os resultados maternos e perinatais (AMORIM et al., 2021).

Desse modo, percebe- se que a assistência pré-natal durante a pandemia é de extrema importância para as mulheres em período gravídico enfrentarem esse período com uma maior segurança, além disso, os serviços de saúde devem contar com uma equipe multiprofissional para proporcionar à gestante toda assistência necessária, esclarecendo dúvidas, alertando sobre os fatores de risco da Covid-19, orientando sobre as formas de prevenção, realizando consultas e, se caso houver alguma gestante infectada, evitar possíveis agravos.

\section{Assistência perinatal}

O assistencialmente perinatal abrange de forma completa o binômio mãefilho, compreendendo todo período gravídico, parto, nascimento, puerpério e atenção neonatal. Nesse processo de cuidados, é necessário o envolvimento de uma equipe multiprofissional, efetuando um atendimento adequado e qualificado (OLIVEIRA, 2021).

A simultaneidade da gravidez/puerpério e infecção por Covid-19 institui muitos obstáculos. Mulheres grávidas devem ser monitorizadas e, se houver casos positivados para Covid-19, tanto a mãe como o neonato necessitam ser 
acompanhados. Menciona- se que o parto cesariano e o isolamento com separação mãe e filho, sem o aleitamento materno, só deve ocorrer caso a mãe ou a criança apresente um quadro clínico grave. Dada essa exceção, a via de parto vaginal deve ser priorizada e incentivada e a amamentação mantida - com a asseguração de todos os cuidados protocolados (CARDOSO, 2021).

Segundo Amorim (2021), a transmissão perinatal pode, teoricamente, suceder- se por meio de micropartículas de secreção respiratória transportadas pelo ar, gotículas maternas ou da equipe dos profissionais presentes durante o parto, ou através do canal de parto ou no decorrer da amamentação - contudo, não existem evidências concretas sobre a ocorrência de infecção de Covid-19 por meio do aleitamento ou pela secreção vaginal. Sendo assim, se a mulher tiver condições e desejar, todas as diretrizes e recomendações devem ser mantidas.

O estudo realizado por Estrela (2020), aponta que gestantes infectadas por Sars-Cov-2 que evoluem para um quadro grave e apresentam alguma comorbidade, na maioria dos casos, são submetidas ao parto cesariano de emergência ou parto vaginal prematuro, trazendo riscos que podem levar a morte materna e neonatal.

De acordo com Stofel (2021), as descobertas científicas precisam ser adeptas à realidade social de cada região. E faz- se indispensável regulamentar e organizar a rede de atenção em saúde materna e infantil, de forma que o serviço chegue a todos com acessibilidade, qualificação, segurança e dando seguimento a esses cuidados para mulheres e recém-nascidos acometidos ou não pela Covid- 19.

\section{CONCLUSÃO}

Tendo em vista a exposição do estudo, sabe- se que a mulher gestante passa por muitas transformações fisiológicas para gerar seu filho e a realização de uma assistência pré-natal qualificada é extremamente necessária para redução de riscos e prevenção a patologias durante a gestação. A importância dessa assistência intensificou- se ainda mais com a pandemia de Covid-19, onde os profissionais de 
saúde tiveram que se reinventar para proporcionar um serviço de qualidade às pacientes e garantir uma experiência gestacional agradável e segura.

O cuidado pré-natal é extremamente significante para a saúde materna, fetal e perinatal, e na luta contra a pandemia, a assistência qualificada ajuda a fortalecer o cuidado e as medidas preventivas para mulheres durante a gravidez para evitar a contaminação por COVID- 19, demonstrando a relevância de um atendimento prénatal capacitado e enfatizando a importância dos profissionais de saúde e da atenção primária.

\section{REFERÊNCIAS BIBLIOGRÁFICAS}

ALMEIDA, Milene de Oliveira; PORTUGAL, Thainá Magalhães; ASSIS, Thais Josy Castro Freire de. Gestantes e COVID-19: isolamento como fator de impacto físico e psíquico. Rev. Bras. Saude Mater. Infant. Recife, v. 20, n. 2, p. 599-602, junho 2020. Disponível em: http://www.scielo.br/scielo.php?script=sci_arttext\&pid=S1519-

38292020000200599\&lng=en\&nrm=iso.

AMORIM, Melania Maria Ramos et al. COVID-19 e Gravidez. Revista Brasileira de Saúde Materno Infantil [online]. 2021, v. 21, n. pp. 337-353. Disponível em: https://doi.org/10.1590/1806-9304202100S200002.

BRASIL. Humanização do parto Humanização no Pré-natal e nascimento. Brasília (DF): Ministério da Saúde; 2002.

BRASIL. Manual de Recomendações para a Assistência À Gestante e Puérpera frente à Pandemia de Covid-19. Brasília (DF): Ministério da Saúde; 2020.

CARDOSO, Pollyanna Costa et al. Saúde materno-infantil no contexto da pandemia de COVID19: evidências, recomendações e desafios.Revista Brasileira de Saúde Materno Infantil [online]. 2021, v. 21, n.pp. 213-220. Disponível em: https://doi.org/10.1590/1806$9304202100 S 100011$.

ESPOSTI, Carolina Dutra Degli et al. Desigualdades sociais e geográficas no desempenho da assistência pré-natal de uma Região Metropolitana do Brasil. Ciência \& Saúde Coletiva [online]. v. 25, n. 5, pp. 1735-1750. Disponível em: https://doi.org/10.1590/141381232020255.32852019 .

ESTRELA, FERNANDA MATHEUS et al. Gestantes no contexto da pandemia da Covid-19: reflexões e desafios. Physis. Rio de Janeiro, v. 30, n. 2, e300215, 2020. Disponível em: http://www.scielo.br/scielo.php?script=sci_arttext\&pid=S0103-

$73312020000200314 \& \operatorname{lng}=$ en\&nrm=iso.

LEAL, Maria do Carmo et al. Prenatalcare in theBrazilianpublichealthservices. Revista de Saúde Pública [online]. v. 54, 08 . Disponível em: https://doi.org/10.11606/s15188787.2020054001458.

LIVRAMENTO, Débora do Vale Pereira do et al. Percepções de gestantes acerca do cuidado 
pré-natal na atenção primária à saúde. Rev. Gaúcha Enferm. Porto Alegre, v. 40, e20180211,2019. Disponível em: http://www.scielo.br/scielo.php?script=sci_arttext\&pid=S1983$14472019000100420 \& \operatorname{lng}=e n \& n r m=i s o$.

MASCARENHAS, Victor Hugo Alves et al. COVID-19 e a produção de conhecimento sobre as recomendações na gravidez: revisão de escopo. Rev. Latino-Am. Enfermagem, Ribeirão Preto, v. 28, e3348, 2020. Disponível em: http://www.scielo.br/scielo.php?script=sci_arttext\&pid=S0104$11692020000100606 \& \operatorname{lng}=$ en\&nrm=iso.

MEDEIROS, Fabiana Fontana et al. Acompanhamento pré-natal da gestação de alto risco no serviço público. Rev. Bras. Enferm., Brasília, v. 72, supl. 3, p. 204-211, dezembro 2019. Disponível em: http://www.scielo.br/scielo.php?script=sci_arttext\&pid=S0034$71672019000900204 \& \operatorname{lng}=$ en\&nrm=iso.

MENDES, Rosemar Barbosa et al. Avaliação da qualidade do pré-natal a partir das recomendações do Programa de Humanização no Pré-natal e Nascimento. Ciênc. saúde coletiva, Rio de Janeiro, v. 25, n. 3, p. 793-804, março 2020. Disponível em: http://www.scielo.br/scielo.php?script=sci_arttext\&pid=S1413-

$81232020000300793 \& \operatorname{lng}=$ en\&nrm=iso.

OLIVEIRA, Maysa Arlany de et al. Recomendações para cuidados perinatais no contexto da pandemia COVID-19. Revista Brasileira de Saúde Materno Infantil [online]. 2021, v. 21, n.pp. 65-75. Disponível em: https://doi.org/10.1590/1806-9304202100S100004.

PEREIRA, Elsa. Gravidez. Saúdebemestar, [S. I.], p. 1-1, 2 nov. 2020. Disponível em: https://www.saudebemestar.pt/pt/clinica/ginecologia/gravidez/. Acesso em: 26 nov. 2021.

SILVA, Fernanda Loureiro, Russo, Jane e Nucci, Marina. Gravidez, parto e puerpério na pandemia: os múltiplos sentidos do risco. Horizontes Antropológicos [online]. 2021, v. 27, n. 59, pp. 245-265. Disponível em: https://doi.org/10.1590/S0104-71832021000100013.

SILVA, Luzenilda Sabina de et al., Análise das mudanças fisiológicas durante a gestação: desvendando mitos. Revista Eletrônica Faculdade Montes Belos, v. 8, n. 2, 2015.

STOFEL, Natália Sevilha et al. Assistência perinatal na pandemia COVID-19: análise de diretrizes e protocolos brasileiros. Revista Brasileira de Saúde Materno Infantil [online]. 2021, v. 21, n.pp. 89-98. Disponível em: https://doi.org/10.1590/1806-9304202100\$100005. 